\title{
Group Selection
}

\section{Andy Gardner}

University of St Andrews

Introduction

General Overviews

Books

Papers

Journals

Origins of the Group Selection Controversy

Theoretical Approaches

Overview

Population Genetics

Price-Hamilton

Quantitative Genetics

Numerical Simulation

Empirical Studies

Relation to Kin Selection

General

Sex Allocation

Species Selection

Group Adaptation and the Superorganism

Major Transitions in Evolution

Shifting Balance

Cultural Group Selection

\section{Introduction}

The basic idea of group selection theory is that the logic of natural selection acting at the level of individual organisms can also be applied to the level of whole groups of organisms. Quite what this means and whether it is a useful way of thinking about the biological world remains very controversial. Indeed, the development of the group selection literature has been driven as much by confusion and misstep as it has by informed reasoning. Although careful and considered foundations were laid in the $19^{\text {th }}$ Century by Charles Darwin, his contributions to the theory of group - or multilevel - selection were largely ignored and, in the $20^{\text {th }}$ Century, gave way to a naïve view that run-of-the-mill natural selection leads inevitably to adaptation for the good of the species as a whole. Since the 1960's progress has proceeded in fits and starts, with a gradual consensus building that adaptation at the level of whole populations requires that selection has been acting at a between-population level, and that adaptation at the level of whole populations tends to be eroded by the action of within-population selection. This development in understanding has led to an interest in conceptualizing and quantifying the action of selection at the group level in a range of theoretical and empirical scenarios. However, no consensus has emerged as to how group selection and related concepts are to be formally defined. Accordingly, the theoretical literature is characterised by repeated returns to first principles and repeated reinventing of the wheel, rather than cumulative and collaborative progress. And this failure of theory has led to the empirical literature being very patchy, with some very nice studies in the laboratory and in the field being motivated and conducted within a multilevel selection framework, but little sustained progress through long-term interplay of theoretical and empirical research. As a research programme, group selection has certainly enjoyed much less success than its competitor the theory of kin selection - which describes exactly the same phenomena in an alternative but equivalent mathematical and conceptual language, and has been shown to have enormous scientific utility in relation to biological topics as diverse as sex ratios, parasite virulence and the evolution of altruism. But group selection does logically appear to provide a superior conceptual framework for understanding the evolution of group-level adaptation and, hence, major transitions in individuality 
(e.g. from unicells, to multicellular animals, to eusocial insect "superorganisms"), and perhaps also the evolution of human culture which, if it is Darwinian at all, may be best conceptualised as being a property of social groups and institutions than of individual persons. Moreover, group selection is of strong interest to philosophers and historians of science, and biologists of a philosophical and historical bent.

\section{General Overviews}

The literature on group selection has been inordinately focused on simply establishing what group selection is and whether it plays any meaningful role in the natural world. Multiple overviews have been published over the last 60 years, often in book form but also as influential review papers.

\section{Books}

There are no textbooks as such on the topic of group selection, but perhaps the best entry point into the literature remains Williams's 1966 book-length attack on the lazy group-selectionist thinking that had taken root in evolutionary biology during the first half of the $20^{\text {th }}$ Century. Although theory and concepts have developed somewhat in the ensuing 50 years, along with the understanding that the action of selection at levels of organisation higher than the individual need not be very weak, the book does largely hold up today, and it highlights how stultifying that uncritical group selectionism had been: obscuring and explaining away fascinating social behaviours, rather than opening them up to informative study. A more contemporary overview of the topic is provided by Okasha 2006, an excellent - though rather more formal - theoretical and philosophical account of the diversity of thinking and approaches to the topic that, remarkably, manages to set down definitions and schemes of classification that appear to be endorsed by almost every researcher currently active on this topic. Okasha 2006 owes a clear debt to Lloyd 1988, which is also very lucid and philosophically focused, albeit now somewhat out of date. Sober \& Wilson's 1998 book makes an impassioned appeal for a return to group-selection thinking, arguing that whilst Williams was largely correct he went too far in rejecting a potentially useful means of thinking about social evolution, and explores potentially useful applications of the theory to human evolution. Finally, a selection of edited volumes provide snapshots of the diversity of thinking on group selection at different times: Williams 1971 collects together a number of historical papers; Keller 1999 features contributions from a number of biologists with a notable behavioural-ecology flavour and, unusually, a strong emphasis on the interplay of theory and empiricism; and Bouchard \& Huneman 2013 focuses on the particular issue of groups as individuals, with a mostly theoretical and philosophical emphasis.

Williams, G. C. 1966. Adaptation and natural selection. Princeton, NJ: Princeton University Press. This landmark book ended the lazy group-selectionist thinking that had characterised the first half of the $20^{\text {th }}$ Century. Although theory and concepts have developed a great deal since the 1960 's, this remains perhaps the best entrypoint into the literature.

Okasha, S. 2006. Evolution and the levels of selection. Oxford, UK: Oxford University Press.

A comprehensive overview of the theory of group selection and related topics, from a strongly-philosophical viewpoint, that has been lauded by researchers across the full breadth of this very contentious topic. Another excellent entrypoint into the literature.

Lloyd, E. 1988. The structure and confirmation of evolutionary theory. Westport, NY: Greenwood Press.

A lucid account of evolutionary theory from a philosophy of biology perspective, with a strong focus on group selection and associated themes, including species selection. Lloyd takes a semantic (model-based) approach to evolutionary theory, to make sense of these controversies. 
Sober, E. \& Wilson, D. S. 1998. Unto others: the evolution and psychology of unselfish behaviour. Cambridge, MA: Harvard University Press.

An overview of theory on the evolution of altruism, with special application to humans and the humanities. Sober and Wilson highlight the equivalence of kin selection and group selection, but argue strongly for the group selectionist approach, mainly on philosophical grounds.

Williams, G. C. 1971. Group selection. Chicago, IL: Aldine Atherton.

A volume of collected papers on group selection and related themes, by several different authors, assembled in order to give "a candid view of a live issue". A useful overview of the group selection debate as it existed in the middle years of the $20^{\text {th }}$ Century.

Keller, L. 1999. Levels of selection in evolution. Princeton, NJ: Princeton University Press.

An edited volume on levels of selection in evolutionary biology, with contributions from leading experts across the field of social evolution - with a distinctly behavioural-ecological flavour. Notable for its strong focus on the interplay between theoretical and empirical research.

Bouchard, F. \& Huneman, P. 2013. From groups to individuals: evolution and emerging individuality. Cambridge, MA: MIT Press.

A recent, edited volume with contributions from philosophers and biologists, looking past the group selection controversy and tackling themes of adaptation, individuality and complexity in a multilevel selection setting.

\section{Papers}

The topic of group selection has been reviewed and re-reviewed with regularity over the last 40 years. Much of the content of these reviews remains very repetitive, but incremental advances have been made along the way, and it is interesting to see how emphases have changed over the decades. Maynard Smith 1976 was very influential at the time but, in highlighting what he saw as a key empirical distinction between kin selection and group selection, it is somewhat at variance with the largely-held contemporary view that kin selection and group selection theories are formally equivalent. Alexander \& Borgia 1978 frames the group selection debate within the wider topic of levels of selection, from the gene level to the species level, highlighting the logical and biological similarities at each level of organisation. Wilson's 1983 paper provides an early overview of theory relating to the more modern conception of group selection as occurring between smaller groupings of sociallyinteracting individuals as opposed to whole populations or species. Damuth \& Heisler 1988 highlights that different researchers often have in mind different conceptions of group selection - which they termed "multilevel selection 1" versus "multilevel selection 2", concerning whether the focus of attention is ultimately on the individual or on the group - and that much of the controversy over group selection stems from these researchers talking past each other as opposed to any empirically substantive disagreement, a view also promulgated in Okasha 2001. Wilson \& Wilson 2007 more forcefully urges a rethink of the study of social evolution, arguing that its foundations should be built upon the theory of group selection. Leigh's 2010 paper provides a more recent overview, and in particular emphasises the pre-history of the group selection controversy, i.e. prior to the 1960s.

Maynard Smith, J. 1976. Group selection. The Quarterly Review of Biology 51: 277-283.

A review of group selection theory, highlighting that group selection occurs and question the extent to which it contributes to evolutionary change, and urging for a distinction between group selection and kin selection on the basis that the former requires group structure whilst the latter does not.

Alexander, R. D. \& Borgia, G. 1978. Group selection, altruism, and the levels of organization of life. Annual Reviews of Ecology \& Systematics 9: 449-474. 
A comprehensive discussion of group selection and related concepts including units of selection, emergence and species selection. This paper reviews the results of theoretical models as well as empirical evidence, and emphasises similarities across multiple levels of biological organization.

Wilson, D. S. 1983. The group selection controversy: history and current status. Annual Review of Ecology and Systematics 14: 159-187.

A review of mathematical models and verbal accounts of group selection spanning the history of the topic, emphasising the confused nature of the literature and reinterpreting kin-selection results using a group selection approach, serving to illustrate the equivalence of approaches.

Damuth, J. \& Heisler, I. L. 1988. Alternative formulations of multilevel selection. Biology \& Philosophy 3: $407-430$.

Damuth and Heisler suggest that progress on multilevel selection has been slowed by a failure to recognise the distinction between multilevel selection 1 - in which the focus is on the individual - versus multilevel selection 2 - in which the focus is on the group.

Okasha, S. 2001. Why won't the group selection controversy go away? British Journal for the Philosophy of Science 52: 25-50.

A philosophical overview of some aspects of the group selection controversy, characterising this in terms of the question of whether natural selection can operate at the level of the group, and concluding that different people have rather different interpretations of what the term "group selection" means.

Wilson, D. S. \& Wilson, E. O. 2007. Rethinking the theoretical foundation of sociobiology. The Quarterly Review of Biology 82: 327-348.

An impassioned plea for the field of social evolution theory to rethink it's rejection of group selection, with a review of history, theory and empiricism and application of group-selection thinking to eusociality and human evolution.

Leigh, E. G. 2010. The group selection controversy. Journal of Evolutionary Biology 23: 6-19. Reviews the development of thinking on the topic of group selection, including the "prehistory" leading up to the controversy of the 1960s. Emphasises the equivalence of kin selection and group selection, as these terms are currently understood, and the usefulness of having alternative conceptualizations.

\section{Journals}

The often sensationalist nature of the group selection controversy means that key papers are often published in the highest-impact journals, including the multidisciplinary publications ${ }^{* *}$ Nature ${ }^{* *}$, ${ }^{* *}$ Science ${ }^{* *}$ and ${ }^{* *}$ Proceedings of the National Academy of Sciences of the USA ${ }^{* *}$. The key discipline in which this topic has originated and been developed is evolutionary biology, and in particular those parts of the field that have placed an emphasis on ecology rather than simply genetics. Accordingly, ${ }^{* *}$ Trends in Ecology and Evolution ${ }^{* *}$ (a review journal) and the society journals ${ }^{* *}$ Evolution ${ }^{* *}$, ${ }^{* *}$ The American Naturalist **and ** Journal of Evolutionary Biology ${ }^{* *}$ tend to be the outlets for much work on this topic. However, the philosophical interest in group selection has led a more recent growth in the number of relevant papers published in journals like ${ }^{* *}$ Biology \& Philosophy ${ }^{* *}$, as well as those serving other disciplines within the social sciences.

\section{${ }^{*}$ Nature [www.nature.com/nature] ${ }^{*}$}

Published by the Nature Publishing Group, this high-impact journal covers all branches of science. The high profile of the group selection debate means that many papers on this topic 
have been published here, although typically these fan the flames of controversy rather than providing resolution.

*Science [www.sciencemag.org] ${ }^{*}$

Published by the American Association for the Advancement of Science, this high-impact journal covers all branches of science. As with Nature, the high profile of the group selection debate means that many papers on this topic have been published here.

*Proceedings of the National Academy of Sciences of the USA [www.pnas.org] ${ }^{*}$

Published by the United States National Academy of Sciences, this high-impact journal covers all branches of science. As with Nature and Science, the high profile of the group selection debate means that many papers on this topic have been published here.

*Trends in Ecology and Evolution [http://www.cell.com/trends/ecology-evolution/home] Published by Cell Press, this journal publishes review papers on evolutionary biology and ecology, including Review, Opinion, Letters, Science \& Society, Spotlight, Book Review and Forum papers.

${ }^{*}$ Evolution [http://onlinelibrary.wiley.com/journal/10.1111/(ISSN)1558-5646] ${ }^{*}$

Published by Wiley on behalf of the Socity for the Study of Evolution, this journal publishes papers on evolutionary biology, including Original Articles, Perspectives, Brief Communications, Technical Comments, Book Reviews, Outlook on Evolution and Society articles and Commentaries.

*The American Naturalist [http://www.press.uchicago.edu/ucp/journals/journal/an.html]" Published by University of Chicago Press on behalf of the American Society of Naturalists, this journal publishes papers in evolutionary biology and ecology, with a notable theoretical slant, including Articles, Notes, Syntheses, Perspectives and Natural History Miscellany.

*Journal of Evolutionary Biology [http://onlinelibrary.wiley.com/journal/10.1111/(ISSN)1420-9101] Published by Wiley on behalf of the European Society for Evolutionary Biology, this journal publishes papers in evolutionary biology, with an emphasis on interplay between theory and empiricism, including Research Articles, Short Communications, Mini-Reviews and Target Reviews.

${ }^{*}$ Biology and Philosophy [http://link.springer.com/journal/10539] ${ }^{*}$

Published by Springer, this journal publishes philosophy of biology papers, including Original Papers, Review Essays, Book Reviews and Brief Communications. A great deal of recent discourse on group selection can be found here and in other philosophy journals.

\section{Origins of the Group Selection Controversy}

The first glimmerings of group selection theory appear in Darwin 1859 , in the context of a discussion about sterile insect works. Although the passage has a predominantly kin selection flavour, some of the wording hints at a group selectionist outlook. Darwin wrote more explicitly about group selection in his 1871 book, in relation to intergroup conflict driving the evolution of selflessness in humans. Again, here the distinction between group selection and kin selection is blurred by his emphasis on consanguinity between group mates as providing an incentive for selflessness in the context of social groups struggling for existence - in line with modern understanding that the two theories are empirically equivalent. Unfortunately, these ideas appear to have been largely ignored by influential writers in the first half of the $20^{\text {th }}$ Century who were of the view that run-of-the-mill natural selection would inevitably result in adaptation for the good of the species, and that individually-injurious adaptations (such as altruism) could be explained away on this basis. Such thinking is abundantly 
illustrated by Lorenz's 1963 book, which is by no means exceptional for its time. The 1958 edition of Fisher 1999 included a passage making explicit that between-species selection is required for species-level adaptation and emphasising that, although logically valid as an evolutionary process, this is likely to be very weak. Wynne-Edwards 1962 recognised that higher levels of selection would often be opposed by selection acting at lower levels, but incorrectly asserted that selection at the higher level must always prevail. Williams 1966 provided a book-level treatment of the problem, bringing Fisher's insights to a much wider audience, and drawing a close to the lazy group selectionism of the first half of the $20^{\text {th }}$ Century. Wilson 1980 salvaged a defensible theory of group selection from the wreckage, and argued that although Williams' central point was correct, there is value in thinking about levels of selection higher than the individual. Borrello 2010 provides an overview of the origins of the controversy, with a focus on Wynne-Edwards' life and work.

Darwin, C. R. 1859. On the origin of species by means of natural selection, or the preservation of favoured races in the struggle for life. London, UK: John Murrary.

Chapter 7 includes a discussion of how natural selection can adapt sterile workers to the tasks they perform in social insect colonies. Darwin's explanation, inspired by breeding practices from agriculture, is primarily of a kin-selection flavour, but his wording sometimes suggests a group-selection perspective. [Full text available *online[http://darwinonline.org.uk $]^{*}$

Darwin, C. R. 1871. The descent of man, and selection in relation to sex. London, UK: John Murrary. Chapter 5 considers the evolution of selflessness to the disadvantage of the individual relative to his group mates but nevertheless favoured owing to the improved success of his group in its struggle against other groups. Although explicitly a group-selection argument, Darwin also emphasises consanguinity as providing motivation for group-beneficial behaviour. [Full text available *online[http://darwin-online.org.uk*]

Lorenz, K. 2002. On aggression. London, UK: Routledge.

Published in German in 1963, Lorenz's book explicitly couches the evolution of social behaviour in terms of benefit to the species, without any recognition that this is puzzling from the individual's perspective. This way of thinking seems to be condoned by Julian Huxley in the short Foreword.

Fisher, R. A. 1999. The genetical theory of natural selection: a complete variorum edition. Oxford, UK: Oxford University Press.

A section on "The benefit of the species" appears in the 1958 edition, in which Fisher explains that natural selection acting within species cannot lead to adaptation for the good of the species. Fisher notes that between-species selection can occur, but with negligible impact upon adaptation. Interestingly, Fisher did suggest a possible role for population-level selection in providing an evolutionary advantage for sexual reproduction, in the 1930 edition of his book.

Wynne-Edwards, V. C. 1962. Animal dispersion in relation to social behaviour. Edinburgh, UK: Oliver \& Boyd.

A more careful - but flawed - account of social behaviour framed in terms of the benefit to group or species, acknowledging that the evolutionary interests of different levels of biological organization will often come into conflict, but incorrectly arguing that the interests of the higher levels will always prevail.

Williams, G. C. 1966. Adaptation and natural selection. Princeton, NJ: Princeton University Press. In this landmark book, Williams reiterated Fisher's point that only by selection acting between groups or higher levels of biological organization can adaptations for the benefit of the group 
or species arise. This book more or less banished lazy group-selectionist thinking from evolutionary biology.

Wilson, D. S. 1980. The natural selection of populations and communities. Menlo Park, CA: Benjamin/Cummings.

An early book-length articulation of the new theory of group selection, tempered by Williams' critique, recognising the power of lower levels of selection and focusing on group selection's quantitative impact upon evolution rather than adaptationst for the benefit of groups or species.

Borrello, M. E. 2010. Evolutionary restraints: the contentious history of group selection. Chicago, IL: University of Chicago Press.

An account of the group selection controversy by a historian of biology, with a strong biographical focus on Wynne-Edwards, and rather sympathetic to his views of adaptation.

\section{Theoretical Approaches}

A diversity of theoretical approaches have been employed in relation to the study of group selection, including population genetics, the Price-Hamilton selection-covariance approach, quantitative genetics and numerical simulations.

\section{Overview}

A diversity of theoretical approaches have been employed in relation to the study of group selection. These grew out of population genetics theory, which provides the gold standard for the formalization of evolutionary arguments, but have subsequently taken different directions. Perhaps the main formalism for thinking about group selection is that which has arisen directly from Price's equation, but competing views of group selection have also emerged from the study of quantitative genetics.

Analytical theory of group selection can only get researchers so far, and often numerical simulations are employed where the mathematics has become too tricky. Maynard Smith 1976 provides a review of models that had been purported to be of group selection, and argues that group selection is either weak or absent in those models. Wade 1978 argues that typical simplifying assumptions lead to the strength of group selection being underestimated and that more realistic assumptions help to bolster the impact of group selection on modelled outcomes. Uyenoyama \& Feldman 1980 reviews models of group selection and attempts to organise them within a formal, population genetics framework. Okasha 2004 discusses the pros and cons of two distinct modelling approaches for group selection, from the Pricean versus quantitative genetics traditions.

Maynard Smith, J. 1976. Group selection. The Quarterly Review of Biology 51: 277-283.

A review of models of group selection, focused on the issue of how strongly group selection may contribute to adaptation and distinguishing between models involving discrete groups with differential extinction versus those that do not (and urging that the latter should be viewed in terms of kin selection).

Wade, M. J. 1978. A critical review of the models of group selection. The Quarterly Review of Biology 53: 101-114.

Wade reviews a number of theoretical models of group selection and suggests that their simplifying assumptions - e.g. number of loci, size and form of social grouping, patterns of migration - will tend to underestimate the scope for group selection to contribute to evolutionary change.

Uyenoyama, M. \& Feldman, M. W. 1980. Theories of kin and group selection: a population genetics perspective. Theoretical Population Biology 17: 380-414. 
This paper includes an overview of models of group selection, from a population genetics perspective, and a discussion of Wright's "shifting balance" theory of evolutionary adaptation.

Okasha, S. 2004. Multi-level selection, covariance and contextual analysis. British Journal for the Philosophy of Science 55: 481-504.

A philosophical overview of the Price-Hamilton versus contextual-analysis approaches to multilevel selection, suggesting that sometimes one and sometimes the other better relates to the intuitive notion of group selection, depending on scenario, but overall finding in favour of the latter approach.

\section{Population Genetics}

Population genetics provides the gold standard for formalizing evolutionary theory. If an evolutionary theory cannot be expressed in such terms, then its validity must be seriously questioned. (Extension of population genetics beyond genetical inheritance in the strict sense is discussed in relation Cultural Group Selection section, below.) However, whilst population genetical methodology may be used to develop models of particular evolving populations within which group selection is acting, these may be relatively unhelpful in terms of providing general definitions of group selection and other evolutionary forces. A classic population genetics model of group selection was developed in Haldane 1932, which sought to show how human altruism may be favoured in the context of a "tribe splitting" demography. The model proved to difficult to analyse fully, but the result was confirmed by numerical simulation decades later (see the *Numerical Simulations * section). A different group selection model, for the evolution of altruistic helping in a population characterised by a low rate of dispersal between social groups, was developed by Wright in a 1945 book review. Again, this was too complex to solve but later yielded to numerical simulations (see the *Numerical Simulations* section), with the surprising result that a low rate of dispersal does not, in fact, promote the evolution of helping. Maynard Smith's 1964 paper introduced the famous "haystack model" of group selection, as a means of estimating the quantitative influence of group selection in a plausible setting. Wilson 1975 develops population genetics models of group selection to articulate and formalise the idea of "trait groups" (i.e. social groups) as units of selection.

Haldane, J. B. S. 1990. The causes of evolution. Princeton, NJ: Princeton Science Library. The Appendix of Haldane's landmark book, first published in 1932, includes an incompletelyanalysed model in which periodic splitting of human tribes generates heritable variation in altruism at the between-tribe level and, hence, allows for the possibility for between-group selection for altruism.

Wright, S. 1945. Tempo and mode in evolution: a critical review. Ecology 26: 415-419.

In this book review, Wright presented an incompletely-analysed model of altruism evolving in a structured population, modulated by selection acting within and between groups, and suggested that limited dispersal between groups would promote the evolution of altruism.

Maynard Smith, J. 1964. Group selection and kin selection. Nature 201: 1145-1147.

This paper introduced the "haystack" model of group selection, in which a single pregnant mouse colonises a haystack and produces a large number of generations of descendants who then disperse, to illustrate that selection may favour group-beneficial behaviours only in species with unusual biology.

Wilson, D. S. 1975. A theory of group selection. Proceedings of the National Academy of Sciences of the USA 72: 143-146.

This paper introduces the "trait group" sense of group selection, in which even temporary groupings of socially interacting individuals that are formed and dissolved within the 
individual's lifetime is considered a unit of selection, and investigates conditions for the evolution of altruism in such contexts.

\section{Price-Hamilton}

The Price-Hamilton approach to defining and quantifying group selection emerges directly from Price's equation of natural selection. Price first published his equation in 1970, in a strictly evolutionary genetical context, but subsequently extended and generalised the equation for application beyond strictly genetical inheritance in a paper in 1972. This latter paper included a section on group selection, in which he showed how the total action of natural selection can be readily decomposed into additve within-group and between-group components. This approach to group selection was later elaborated by Hamilton in 1975, and he made clear how it relates to the exactly equivalent theory of kin selection. Wade 1985 applies the Price-Hamilton formalism to models incorporating soft versus hard selection (i.e. density dependent regulation before versus after dispersal), showing how these modulate the intensity of group selection. Frank 2013 conceptualises the within-group versus between-group partition in terms of Price's earlier selection versus transmission partition of evolutionary forces, framing group selection within a broader synthesis including, for example, mutation-selection balance. Gardner 2015synthesises the within-group versus between-group partition with Price's earlier application of his equation to class-structured populations, formalising the application of group selection theory to models in which individuals vary in their sex, age, caste and ploidy.

Price, G. R. 1972. Extension of covariance selection mathematics. Annals of Human Genetics 35: 485-490.

This paper presents a number of extensions and generalizations of the Price equation. A section on group selection showes how natural selection can be decomposed into a simple sum of between-group and within-group components, for potentially multiple levels of selection.

Hamilton, W. D. 1975. "Innate social aptitudes of man: an approach from evolutionary genetics." In Biosocial Anthropology. Edited by R. Fox, 133-153. London, U: Malaby Press.

Hamilton reviewed Price's approach to levels of selection, bringing it to greater attention, and showed how it links with the mathematics of kin selection, providing a first demonstration of the equivalence of these two ways of thinking about social evolution.

Wade, M. J. 1985. Soft selection, hard selection, kin selection and group selection. The American Naturalist 125: 61-73.

An extension of the Price-Hamilton approach to group selection, showing how hard selection versus soft selection modulates the relative intensity of within-group versus between-group selection, and making links with kin selection.

Frank, S. A. 2013. Natural selection III: selection versus transmission and the levels of selection. Journal of Evolutionary Biology 25: 227-243.

An account of the Price-Hamilton approach to multilevel selection framed within the context of the Price equation's selection-transmission partition, providing the basis for a synthesis of ideas including the stochastic corrector model, mutation-selection balance and parasite virulence.

Gardner, A. 2015. The genetical theory of multilevel selection. Journal of Evolutionary Biology 28: 305-319.

An extension of the Price-Hamilton approach to multilevel selection in the context of classstructured populations, showing that the theory of reproductive value resolves ambiguities over the definition of group fitness and the meaning of group selection. 


\section{Quantitative genetics}

A different family of approaches to capturing the action of group selection has emerged from quantitative genetics, based upon the influential work of Lande \& Arnold on multivariate "selectiongradient analysis". Heisler \& Damuth 1987 combines the selection-gradient approach with a research tool developed in the social sciences, resulting in the "contextual analysis" approach to group selection. In contrast to the Price-Hamilton approach, in which group selection is conceived as being driven by differences in group fitness, the contextual-analysis approach conceives group selection as being driven by the impact that a group-level trait has on individual fitness. Goodnight et al. 1992 investigates the action of group selection, so defined, in the context of models of hard versus soft selection, and comes to rather different conclusions from those of Wade's 1985 corresponding analysis for the Price-Hamilton approach (see Price-Hamilton section, above). A different quantitativegenetical approach to group selection has been explored by Bijma and colleagues (Bijma et al. 2007, Bijma \& Wade 2008), including linking multilevel selection to the study of "indirect genetic effects", i.e. when traits beyond simply fitness are modulated by social interaction.

Heisler, I. L. \& Damuth, J. 1987. A method for analyzing selection in hierarchically structured populations. The American Naturalist 130: 582-602.

Working within the selection-gradient analysis research tradition, Heisler and Damuth introduced "contextual analysis" from the social sciences to partion the effects of individual versus group characters in relation to an individual's fitness.

Goodnight, C. J., Schwartz, J. M. \& Stevens, L. 1992. Contextual analysis of models of group selection, soft selection, hard selection, and the evolution of altruism. The American Naturalist 140: 743-761.

An extension of the contextual analysis approach to group selection, showing how hard selection versus soft selection modulates the relative importance of individual versus groupaverage trait in determining the individual's fitness.

Bijma, P., Muir, W. M. \& Van Arendonk, J. A. M. 2007. Multilevel selection 1: quantitative genetics of inheritance and response to selection. Genetics 175: 277-288.

This paper presents a quantitative genetics framework for studying the evolution of social traits in a multilevel selection context, generalizing the earlier work of Griffing and discussing implications for evolutionary biology and agriculture.

Bijma, P. \& Wade, M. J. 2008. The joint effects of kin, multilevel selection and indirect genetic effects on response to genetic selection. Journal of Evolutionary Biology 21: 1175-1188.

A quantitative genetics multilevel selection framework incorporating "indirect genetic effects", i.e. interactions between individuals that affect phenotypic traits in addition to fitness.

\section{Numerical Simulations}

Group selection analysis is often tricky, especially with regards to determining evolutionary outcomes that are modulated by a balance of variation in fitness and heritable trait values at both the withingroup and the between-group levels. For this reason, there is often recourse to numerical simulation methodology, when group selection problems are judged analytically intractable. As with the basic population-genetical approach (see Population Genetics section, above), a numerical simulation model may be interpretable as involving group selection, but it does not directly provide a definition for group selection, and the results obtain in the same way irrespective of how the evolutionary process has been conceptualised. Levin et al. 1974 is an early example, which used numerical simulation data as evidence for the view that group selection can contribute to the evolutionary process but is generally too weak to be of strong relevance. Wilson et al. 1992 revisited Wright's 1945 incomplete analysis of altruism in low-dispersal populations (see Population Genetics section, above) and 
revealed that - in contrast to Wright's suggestion - the rate of dispersal does not affect the condition for a helping allele to be favoured by natural selection. Similarly, Goodnight's 1992 simulation study revisited Haldane's 1932 "tribe-splitting model" of altruism (see Population Genetics section, above), this time confirming the logic of the earlier, incomplete analysis. Avilés 1993 applied a similar tribesplitting (or "budding") model to social spiders, confirming the possible role for group selection to drive the strongly female-biased sex ratios observed in those taxa.

Levin, B. R. \& Kilmer, W. L. 1974. Interdemic selection and the evolution of altruism: a computer simulation study. Evolution 28: 527-545.

A rather early numerical simulation study exploring the potential for group selection to favour genes for altruism that are opposed by selection acting within groups, and concluding that although group selection can favour altruism the usual impact of group selection will be rather weak.

Wilson, D. S., Pollock, G. B. \& Dugatkin, L. A. 1992. Can altruism evolve in purely viscous populations? Evolutionary Ecology 6: 331-341.

A simulation study showing that a reduction in the rate of dispersal of individuals between groups does not promote the evolution of altruism because, whilst reduced dispersal increases heritable variance between groups, it reduces the benefit of altruism in terms of group fitness.

Goodnight, K. F. 1992. The effect of stochastic variation on kin selection in a budding-viscous population. The American Naturalist 140: 1028-1040.

A simulation study of Haldane's tribe-splitting model for the evolution of altruism, conceptualised in terms of both kin and group selection, and confirming that sampling error in the formation of offspring groups from their parens yields between-group variance that fuels group selection.

Avilés, L. 1993. Interdemic selection and the sex ratio: a social spider perspective. The American Naturalist 142: 320-345.

A simulation study, inspired by the biology of social spiders, showing that female-biased sex ratios evolve when groups reproduce by "budding" - i.e. similar to Haldane's (1932) tribesplitting model of human altruism.

\section{Empirical Studies}

The diversity of theoretical methodologies used to define and measure the action of group selection has resulted in an empirical literature that is often internally inconsistent. Whilst some very clever and illuminating empirical studies have been motivated and conducted within a multilevel-selection framework, these typically involve a demonstration that group selection is occurring in a naturalistic setting or, when group selection is artificially applied, that it can yield a significant evolutionary response, or else a proof-of-principle of group-selection methodology in terms of separating out the multivariate causal factors driving differences in fitness or phenotypes or evolutionary trajectories. For example: Wade 1976 and Goodnight 1990 show that artificially-applied group selection yields an evolutionary response in flour beetles; Tsuji 1995 uses contextual analysis to provide quantitative estimates of group-level effects; Muir 1996 shows that artifically-applied selection at the level of whole batches of farmed poultry can lead to improved yield; Aspi et al.'s 2003 study quantifies the impact of group membership on phenotypic traits in campions; Bowles 2006 quantifies the degree of genetic structuring of human populations to assess the relative power of group selection to drive human evolution; Eldakar et al. 2010 shows that female waterstriders flee groups in which males are overly aggressive, putting the brakes on the evolution of sexual conflict; and Pruitt \& Goodnight 2008 shows that a behavioural polymorphism appears to be an important determinant of colony-level fitness in social spiders. That is, at an empirical level, group-selection methodology has often been used as a 
theoretical and conceptual framework for studying group selection, but it remains to have any sustained application beyond the self-referential.

Wade, M. J. 1976. Group selection among laboratory populations of Tribolium. Proceedings of the National Academy of Sciences of the USA 73: 4604-4607.

An experimental study in which strong selection for body size applied to small groups of flour beetles resulted in a rapid evolutionary response.

Goodnight, C. J. 1990. Experimental studies of community evolution I: the response to selection at the community level. Evolution 44: 1614-1624.

An experimental study showing that selection applied at the level of a community comprising two species of flour beetle leads to correlated evolutionary responses between the species, interpreted as coadaptation.

Tsuji, K. 1995. Reproductive conflicts and levels of selection in the ant Pristomyrmex pungens: contextual analysis and partitioning of covariance. The American Naturalist 146: 586-607.

An empirical study that uses a contextual analysis approach to quantify individual and group selection in relation to worker reproduction in a queenless, parthenogetically-reproducing species of ant.

Muir, W. M. 1996. Group selection for adaptation to multiple-hen cages: selection program and direct responses. Poultry Science 75: 447-458.

An experimental study showing that artificial selection applied at the group level leads to improved yield in the context of poultry production, by reducing selection for within-group aggression.

Aspi, J., Jäkäläniemi, A., Tuomi, J. \& Siikamäki, P. 2003. Multilevel phenotypic selection on morphological characters in a metapopulation of Silene tatarica. Evolution 57: 509-517.

An empirical study that uses a contextual analysis approach to quantify individual and group selection in relation to height and stem number in naturally-occurring groups of campions, highlighting that while levels of selection may be in conflict for some traits they need not be for others.

Bowles, S. 2006. Group competition, reproductive levelling, and the evolution of human altruism. Science 314: 1569-1572.

This study uses an interplay of mathematical modelling and empirical estimates of degree of genetic structuring across a number of human populations to assess the importance for between-group competition to have driven the evolution of altruism among early humans.

Eldakar, O. T., Wilson, D. S., Dlugos, M. J. \& Pepper, J. W. 2010. The role of multilevel selection in the evolution of sexual conflict in the water strider Aquarius remigis. Evolution 64: 3183-3189.

A multilevel selection analysis of the fitness effects of male aggression in water striders, revealing that groups with more aggressive males achieve lower mating success, as a consequence of females dispersing away, and that this acts to inhibit the evolution of male aggression.

Pruitt, J. N. \& Goodnight, C. J. 2014. Site-specific group selection drives locally adapted group compositions. Nature 514: 359-362.

An experimental study showing that the survival of artificially-contructed colonies of social spiders depends upon the ratio of docile versus aggressive females, with different environments favouring different ratios, thereby explaining the observed heterogeneity in this ratio in natural populations. 


\section{Relation to Kin Selection}

Notions of group selection were first formulated to make sense of social evolution. Since then, an alternative "kin selection" approach to understanding the same set of biological phenomena has established itself as the conventional approach to the study of social evolution. Throughout much of their respective histories, the theories of group selection and kin selection have often been thought to provide competing hypotheses as to how evolution operates. More recently, a consensus is becoming established that the two theories do not disagree in any substantive way and that they are simply alternative - but equivalent - ways of describing the very same evolutionary processes.

\section{General}

The general consensus is currently that the group-selection approach (i.e. conceiving of natural selection as operating both within and between groups) and the kin-selection approach (i.e. conceiving of natural selection as operating both directly, through the individual's impact upon their own fitness, and indirectly, through the individual's impact on her relatives' fitness) to social evolution are simply different ways of thinking about the very same process. That is, although conceptually distinct, the two methodologies should never yield conflicting predictions in any given scenario, and hence they are not competing scientific hypotheses about how the world works. This insight emerged 40 years ago, and was made clear in Hamilton's 1975 book chapter in which he linked Price's multilevel selection formulation with the mathematics of kin selection, but it has taken some time to become well-known. The studies of Wade 1985 and Queller 1992 relayed this general message to researchers more sympathetic to the group-selection tradition and those more sympathetic to the kinselection tradition, respectively. The message now features in textbooks, such as Rice 2004. Gardner et al. 2007 explores the links between the two theories in the context of multilocus evolution. Lion et al. 2011 provides a relatively recent overview, and van Veelen et al 2012 is a notable example of some researchers continuing to take an opposing view on this. Subsequently, there has been a more nuanced discussion of the senses in which the two theories are similar and the senses in which they are different, and Frank 2013 is an excellent and thoughtful meditation on this topic, framed in terms of causal analysis. Wilson's 2014 recent book on altruism, written for a very general audience, argues that, despite this equivalence, the group-selection approach is nevertheless the better of the two. Kramer \& Meunier's 2016 review article takes a more balanced approach and emphasises the complementarity of kin and group selection outlooks.

Hamilton, W. D. 1975. "Innate social aptitudes of man: an approach from evolutionary genetics." In Biosocial Anthropology. Edited by R. Fox, 133-153. London, UK: Malaby Press.

This book chapter uses Price's equation to show that the kin selection and group selection partitions of natural selection are equivalent.

Wade, M. J. 1985. Soft selection, hard selection, kin selection and group selection. The American Naturalist 125: 61-73.

An extension of the Price-Hamilton approach to group selection, showing how hard selection versus soft selection modulates the relative intensity of within-group versus between-group selection, and making links with kin selection.

Queller, D. C. 1992. Quantitative genetics, inclusive fitness, and group selection. The American Naturalist 139:540-558.

A more detailed investigation of the equivalence of kin selection and group selection results, and the issue of nonadditive fitness effects, based upon the Price equation and an explicitly quantitative genetical approach to social evolution theory.

Rice, S. H. 2004. Evolutionary theory. Sunderland, MA: Sinauer Associates. 
Chapter 10 of this book is dedicated to multilevel selection, and considers in detail its links with inclusive fitness theory, arguing that kin selection is a special form of group selection.

Gardner, A., West, S. A. \& Barton, N. H. 2007. The relation between multilocus population genetics and social evolution theory. The American Naturalist 169: 207-226.

This paper establishes the formal equivalence between kin and group selection in a multilocus context and analyses kin selection and group selection model scenarios using multilocus methodology.

Lion, S., Jansen, V. A. A. \& Day, T. 2011. Evolution in structured populations: beyond the kin versus group debate. Trends in Ecology and Evolution 26: 193-201.

A relatively up to date review of the equivalence of the theories of kin selection and group selection, highlighting the distinction between mathematical methodology and conceptual framework.

van Veelen, M., Garcia, J., Sabelis, M. W. \& Egas, M. 2012. Group selection and inclusive fitness are not equivalent: the Price equation vs models and statistics. Journal of Theoretical Biology 299: 64-80.

This paper provides a notable exception to the growing consensus that there is a formal equivalence between the theories of group selection and kin selection.

Frank, S. A. 2013. Natural selection VII: history and interpretation of kin selection theory. Journal of Evolutionary Biology 26: 1151-1184.

An account of the relationship between kin selection and group selection theories emphasising their differences in terms of causal analysis, and suggesting that kin selection has provided a more useful framework within which to develop scientifically valuable theory.

Wilson, D. S. 2015. Does altruism exist? New Haven, CT: Yale University Press.

A recent book emphasising the equivalence of the theories of kin selection and group selection and arguing for the conceptual superiority of the latter.

Kramer, J. \& Meunier, J. 2016. Kin and multilevel selection in social evolution: a never-ending controversy? F1000Research 5: 776.

A recent review article investigating what is driving the longstanding controversy between group and kin selection and emphasising that the two theories provide complementary rather than competing explanations for social evolutionary phenomena.

\section{Sex Allocation}

As is true for several fundamental issues in the study of social evolution, and Darwinian adaptation more generally, the relationship between group selection and kin selection was first thrashed out and decisively settled - in the context of the study of sex allocation, decades before the rest of the evolutionary biology community caught up. Sex allocation concerns the tradeoff between female and male function, such as the decision of a parent as to how much of their reproductive resources they should invest into producing sons versus daughters. The relevance of sex allocation to group selection, and vice versa, was highlighted by Williams in his 1966 book, when he pointed out that, as females tend to enhance group productivity more than males, group selection should favour a relatively female-biased sex ratio. Accordingly, he took the generally observed equal investment into females and males across most animal and plant species as strong evidence for the weakness of group selection. The following year, Hamilton 1967 opened up the study of sex allocation, and emphasised the abundance of taxa exhibiting female-biased sex ratios. This work was framed in terms of kin selection theory but, in a footnote to the paper, Hamilton made the link with group selection. Colbert's 1981 paper in Nature went further, and pressed the point that the female bias owed to group selection. This elicited a number of commentaries, from Charlesworth and others, in 
1982. Frank's 1986 theoretical study, extending Hamilton's theory of "local mate competition" and framing the results explicitly in terms of group selection, showed comprehensively and decisively that kin selection and group selection theories are simply different ways of describing the same thing. A short book chapter by Maynard Smith 1987 acknowledged the equivalence but emphasised that the resulting sex allocation must be interpreted as an individual-level adaptation rather than an adaptation at the group-level, because it maximizes the individual's (inclusive) fitness and not the group's fitness. Lloyd 1988 provided an overview of this episode, shortly after the dust had settled. West's 2009 book provides a more recent account, and discusses the many developments in the theory of sex allocation that have emerged since then.

Williams, G. C. 1966. Adaptation and natural selection. Princeton, NJ: Princeton University Press. In this book, Williams pointed out that whilst selection acting within well mixed populations favours an even sex ratio, selection at the level of the population often favours a female bias, and that the relatively unbiased sex ratios seen in nature thereby suggest that group selection is relatively unimportant.

Hamilton, W. D. 1967. Extraordinary sex ratios. Science 156: 477-488.

This paper highlights the abundance of female-biased sex ratios in the natural world and explores a number of possible mechanisms for driving these, including a kin selection theory of local mate competition which, in a footnote (\#43), is also conceptualised in terms of selection within and between groups.

Colwell, R. K. 1981. Group selection is implicated in the evolution of female-biased sex ratios. Nature 290: 401-404.

This paper presents a more detailed analysis of local mate competition in terms of the balance of selection acting within and between groups.

Charlesworth, B. C., Toro, M. A., Borgia, G., Wildish, D. J. \& Colwell, R. K. 1982. Female-biased sex ratios. Nature 298: 494-496.

This series of three responses to Colwell (1981), along with Colwell's reply, provides an illustration of the diversity of opinion on how to interpret the theory of local mate competition at that time.

Frank, S. A. 1986. Hierarchical selection theory and sex ratios 1: general solutions for structured populations. Theoretical Population Biology 29: 312-342.

A comprehensive theoretical analysis of sex-ratio evolution using multilevel selection methodology, incorporating local mate competition, local resource competition, asymetric relatedness to sons versus daughters, and Maynard Smith's "haystack" model, highlighting the equivalence to kin selection.

Maynard Smith, J. 1987. Reply to Sober. In The latest on the best: essays on evolution and optimality. Edited by J. Dupré, 147-149. Cambridge, MA: MIT Press.

This very short chapter makes a number of points about group selection, and emphasises that sex ratios modulated by both within-group and between-group selection will not, in general, be optimised for the good of the group and hence do not constitute group-level adaptations.

Lloyd, E. 1988. The structure and confirmation of evolutionary theory. Westport, NY: Greenwood Press.

Pages 90-92 of this book provide an overview of the debate concerning kin selection versus group selection interpretations of female-biased sex ratios that raged through the 1980s. 
West, S. A. 2009. Sex allocation. Princeton, NJ: Princeton University Press.

A relatively recent and comprehensive account of the field of sex allocation, with the history and relative utility of kin selection versus group selection interpretations discussed in Chapter 11.

\section{Species Selection}

Most of the focus of the group-selection literature is on social groups or semi-isolated populations, but its logic has often been applied at the level of whole species or higher taxonomic groupings. Chapter 6 of Lloyd's 1988 book provides perhaps the best entrypoint into this literature. The 1958 edition of Fisher's 1999 book highlighted the logical feasibility of species-level selection and, indeed, pointed out that this would be necessary in order for there to be adaptations that function for the good of the species per se. But Fisher emphasised that the likely impact of selection at this level on the overall evolutionary process would be very weak. Stanley 1975, in contrast, argued that it is a relatively strong force, that will often swamp within-species processes which, as he saw it, were more usually characterised by stasis than directional change. Gould \& Eldredge 1997 on "punctuated equilibria" argued, along similar lines, that most evolutionary change occurs in conjunction with speciation and that differential extinction within clades of closely-related species was the main driver of phenotypic evolution. Gilinksi 1981 suggested that there was evidence for stablizing selection at the species level in relation to shell shape in Archaeogastropoda, and highlighted differential rates of speciation as a possible driver acting alongside differential extinction of species. Charlesworth et al. 1982 offered a strongly opposing view on punctuated equilibrium and species-level selection. More recently, Nunney 1999 has emphasised that species-level selection may do useful work that is unopposed by withinspecies selection, for example in terms of tweaking genetic architecture in ways that reduce the likelihood of occurrence of population-endangering asexuality. Rankin et al. 2015 provides a nice application of the Price-Hamilton approach to multilevel selection being applied at higher-than-usual levels of biological organisation, to estimate the various drivers of mammalian body size evolution.

\section{Lloyd, E. 1988. The structure and confirmation of evolutionary theory. Westport, NY: Greenwood} Press.

Chapter 6 of this book provides a thorough overview of the logic of species selection and its relationship with group selection theory.

Fisher, R. A. 1999. The genetical theory of natural selection: a complete variorum edition. Oxford, UK: Oxford University Press.

A section on "The benefit of the species" appears in the 1958 edition, in which Fisher explains that natural selection acting within species cannot lead to adaptation for the good of the species. Fisher notes that between-species selection can occur, but with negligible impact upon adaptation.

Stanley, S. M. 1975. A theory of evolution above the species level. Proceedings of the National Academy of Sciences of the USA 72: 646-650.

This paper argues that species-level selection acting in the context of speciation will be a strong evolutionary force relative to selection acting at a within-species level, and explores how the former effects may be quantified.

Gould, S. J. \& Eldredge, N. 1977. Punctuated equilibria: the tempo and mode of evolution reconsidered. Paleobiology 3: 115-151.

This paper outlines a similar view of evolution in which very rapid change occurs in newlyformed species which then remain more or less unchanged until their extinction, with specieslevel selection operating through differential extinction within clades of closely-related species. 
Gilinsky, N. L. 1981. Stablizing species selection in the Archaeogastropoda. Paleobiology 7: 316-331. A study showing a macroevolutionary tendency for both low-spired and high-spired genera of archaeogastropods to be lost while genera with intermediate spires predominate, interpreted as the work of species selection, and emphasising the importance of species proliferation as well as extinction.

Charlesworth, B., Lande, R. \& Slatkin, M. 1982. A neo-Darwinian commentary on macroevolution. Evolution 36: 474-498.

This paper argues against the punctuated equilibrium view of evolution, and argues that species-level selection is too weak and slow to account for complex adaptation.

Nunney, L. 1999. Lineage selection: natural selection for long-term benefit. In Levels of Selection in Evolution. Edited by L. Keller, 238-252. Princeton, NJ: Princeton University Press.

A discussion of "lineage selection", including the possibility that selection may act at the species level to modify genetic and developmental architecture so as to minimize the appearance of asexual reproduction, thereby contributing to the evolutionary maintenance of sexual reproduction.

Rankin, B. D. et al. 2015. The extended Price equation quantifies species selection on mammalian body size across the Palaeocene/Eocene Thermal Maximum. Proceedings of the Royal Society of London B 282: 20151097.

A recent application of the Price-Hamilton approach to understanding the dwarfing of mammalian taxa during a warming event $\sim 56$ million years ago, indicating that - in contrast to previous suggestions that it promoted dwarfism - species-level selection may actually have favoured larger body sizes.

\section{Group Adaptation and the Superorganism}

The present consensus is that group selection, like kin selection, is ubiquitous in the natural world, and that - depending on how one defines ones groups - it often makes a significant impact upon the evolutionary process. Under this view, group selection is rarely the sole or most important factor in evolution and, accordingly, groups are not usually expected to manifest complex adaptive design in their own right. Yet the old ideas of the superorganism and evolution for the good of the group or species do continue to haunt the group selection literature, and these do play an important role in preventing group selection from being taken seriously by many evolutionary biologists. However, these ideas may have some biological relevance, in some special situations. Wheeler 1911 introduced the concept of the superorganism to the serious study of the social insects, and he made a compelling case for the similarities between the ant nest, on the one hand, and the traditional multicellular organism, on the other hand, in terms of their structures, behaviours and self-maintaining physiologies. Later, Marais 1937 employed the superorganism view as a conceptual framework for the study of termites, likening the termitiary to a single organism. Williams' 1966 book highlighted that, despite the attractiveness of the superorganism view, biological populations cannot generally be thought of in this way, and that selection at the level of the group would be necessary in order for the group to manifest adaptation in its own right. Maynard Smith's 1987 short book chapter added the second requirement that, in addition to there being group selection, group-level adaptation requires that selection at lower levels of biological organisation essentially be abolished because, otherwise, the adaptations that evolve will not be solely for the good of the group. Seeley 1989 gives the honeybee the superorganism treatment, and argues that this view provides real insights into the biology of these highly social organisms. Sober \& Wilson 1989 argues for a revival of the superorganism concept and develops a theoretical framework for measuring the degree of group adaptation, based on the relative traction that between-group versus within-group selection has on evolutionary adaptation. Gardner \& Grafen 2009 provides a more formal analysis that links the mathematics of multilevel selection dynamics with optimization theory to confirm Williams' and 
Maynard Smith's principles of group-level adaptation (i.e. between-group selection must be present and within-group selection must be absent) and hence yields a more stringent condition for the emergence of the superorganism. Hölldobler \& Wilson 2009 is a gentle, coffee-table book filled with beautiful images of social insects (mostly ants), and framed within the colony-as-organism view.

Wheeler, W. M. 1911. The ant-colony as an organism. Journal of Morphology 22: 307-325.

This paper investigates the ways in which an ant colony resembles a single organism, emphasising such aspects as a separation of germline (reproductives) and somatic (workers) tissues, a clear and coordinated ontogeny, and homeostasis. A classic paper of the social insect literature.

Marais, E. 1973. The soul of the white ant. London, UK: Penguin Books.

Marais, a South African lawyer and naturalist, pioneered the study of termites. First published in Afrikaans in 1937 this book explicitly frames the termitiary as a single "composite animal".

Williams, G. C. 1966. Adaptation and natural selection. Princeton, NJ: Princeton University Press. A key point of this book is that only by the action of group selection can a group evolve adaptations in its own right, such that "for the good of the group" or "for the good of the species" explanations may only be invoked when it is plausible that such higher-level selective mechanisms occur.

Maynard Smith, J. 1987. Reply to Sober. In The latest on the best: essays on evolution and optimality. Edited by J. Dupré, 147-149. Cambridge, MA: MIT Press.

This very short chapter points out that, in addition to the requirement for between-group selection, group-level adaptation also requires that selection within the group be abolished as, otherwise, the phenotype that is favoured will not usually be the one that maximizes group fitness.

Seeley, T. 1989. The honey bee colony as a superorganism. American Scientist 66: 546-553. This paper gives honey bees the superorganism treatment, with an emphasis on division of labour and systems of communication involved in the coordinated functioning of the hive.

Sober, E. \& Wilson, D. S. 1989. Reviving the superorganism. Journal of Theoretical Biology 136: 337356.

This paper outlines an "superorganism continuum" framework in which the relative extent to which group selection has been responsible for shifting a phenotype in the direction of the group's optimum is used as a measure of group adaptation and superorganismality.

Gardner, A. \& Grafen, A. 2009. Capturing the superorganism: a formal theory of group adaptation. Journal of Evolutionary Biology 22: 659-671.

This paper develops formal links between multilevel selection dynamics and group-fitness optimization to confirm Williams' and Maynard Smith's principles of group-level adaptation, i.e. that this requires both group selection and the absence of within-group selection.

Hölldobler, B. \& Wilson, E. O. 2009. The superorganism: the beauty, elegance, and strangeness of insect societies. London, UK: W. W. Norton.

More of a coffee table book than a text book, this showcases the exquisite cooperative adaptations of the social insects - with a major emphasis on the ants - within the superorganism framework, with a tendency to underplay the very interesting conflicts of interests at work within these societies.

\section{Major Transitions in Evolution}


Closely related to the topic of group-level adaptation and superorganisms is the concept of major transitions in evolution - and, in particular, major transitions in individuality. This research theme has grown out of the observation that the interests of the individual and the social group are often in conflict, and yet such conflicts have sometimes been resolved to the extent that the social group takes the appearance of individuality in its own right, such as when unicellular life gives rise to multicellular organisms, and when animal social groups evolve into complex societies with obligate division of reproductive labour. Leigh's 1977 paper frames these ideas in very clear terms, with a focus on the evolution of a unified genome in which the interests of individual genes are aligned owing to the fairness of meiosis. Buss's 1987 book focuses on the evolution of multicellularity and argues that in the details of embryological development we see the fossilized remains of ancient evolutionary conflicts and their resolution. Maynard Smith's 1987 book chapter gathers together several such major transitions and shows the similarities in their logic, highlighting the importance of resolving lower-level conflicts at each step. Maynard Smith \& Szathmáry's 1995 book provides a comprehensive treatment, bringing the major transitions research programme to a much wider audience, and framing it explicitly in terms of levels of selection in conflict. Michod's 2006 study develops a mathematical theory of major transitions involving an exporting of fitness from the lower level to the higher level. Stearns' 2007 perspective article suggests that the human condition may be explicated under the view that our species has stalled during a major transition to eusociality. Calcott \& Sterelny's 2011 edited volume provides a useful overview of recent opinion on the major transitions and levels of selection, with a notably philosophical flavour. Fisher et al.'s 2013 comparative study of origins of multicellularity shows that clonal relatedness - which abolishes within-group selection - has been an important driver of obligate multicellularity and group-level adaptive complexity. Keeling et al 2015 and its associated colloquium papers provide a useful snapshot of current thinking on major transitions in individuality.

Leigh, E. G. 1977. How does selection reconcile individual advantage with the good of the group? Proceedings of the National Academy of Sciences of the USA 74: 4542-4546.

This paper explores how mechanisms that abolish conflicts of interest within social groups may facilitate the evolution of integrated, coordinated adaptation according to the interests of the higher level, with a focus on fair meiosis and the cost of sexual reproduction.

Buss, L. W. 1987. The evolution of individuality. Princeton, NJ: Princeton University Press. This book focuses on the evolution of multicellularity and argues that key aspects of embryogenesis bear the hallmarks of ancient evolutionary conflicts and the mechanisms that have evolved to suppress them.

Maynard Smith, J. 1987. Evolutionary progress and levels of selection. In Evolutionary Progress. Edited by M. H. Nitecki, 219-230. Chicago, IL: University of Chicago Press.

This book chapter defends a particular view of evolutionary progress, in a multilevel selection context, in which mechanisms that abolish selection at lower levels give rise to new units of biological organisation at higher levels.

Maynard Smith, J. \& Szathmáry, E. 1995. The major transitions in evolution. Oxford, UK: Oxford University Press.

This landmark book describes several major transitions in evolution in which there has been a revolution in the way that heritable information is stored and transmitted, including a number of transitions in individuality: from replicator to genome, from cell to multicellular organism, and from organism to society.

Michod, R. E. 2006. The group covariance effect and fitness trade-offs during evolutionary transitions in individuality. Proceedings of the National Academy of Sciences of the USA 103: 9113-9117. 
This paper develops a mathematical theory of major transitions in individuality in which division of labour is associated with an exporting of fitness from the individual to the group level.

Stearns, S. C. 2007. Are we stalled part way through a major evolutionary transition from individual to group? Evolution 61: 2275-2280.

This thought-provoking paper suggests that several aspects of human social behaviour are readily explained on the assumption that our species has begun, but not completed, a major transition to eusociality.

Calcott, B. \& Sterelny, K. 2011. The major transitions in evolution revisited. Cambridge, MA: MIT Press.

This recent edited volume provides a nice overview of current thinking in relation to the major transitions in evolution, with a notably strong philosophical flavour.

Fisher, R. M., Cornwallis, C. K. \& West, S. A. 2013. Group formation, relatedness and the evolution of multicellularity. Current Biology 23: 1120-1125.

This paper reports a comparative study of independent evolutions of multicellularity showing that species in which social groups are clonal (i.e. abolishing within-group selection) are more likely to transition to obligate multicellularity and form more complex groups.

Keeling, P. J., McCutcheon, J. P. \& Doolittle, W. F. 2015. Symbioses becoming permanent: survival of the luckiest. Proceedings of the National Academy of Science, USA 112: 10101-10103.

This introductory article to the "Symbioses becoming permanent: the origins and evolutionary trajectories of organelles" Sackler colloquium, together with the collquium papers that follow it, provide a useful snapshot of current thinking on major transitions in individuality.

\section{Shifting Balance}

Prior to the flare up of the group selection controversy in the 1960s, which was mainly focused on social evolution and the design rationale expected of adaptations emerging from the evolutionary process, a somewhat related controversy arose in the population genetics literature concerning the role for group selection to determine the rate, effectiveness and creativity of evolutionary adaptation. Wright 1932 suggested that, owing to pervasive epistasis, well-mixed populations could become stuck at one of any number of evolutionarily stable points, and that these could greatly vary in adaptiveness (in terms of population productivity). Accordingly, he suggested that adaptive evolution would proceed most rapidly and effectively in large populations that were subdivided into fairly-isolated demes, in which different adaptive explorations could be made and, subsequently, those demes that had hit on the best adaptations could export these to the wider population in a process of inter-demic selection. This "shifting balance" view of adaptive evolution strongly differed from that of Fisher, and fueled the famous, long-running dispute between these two intellectual giants. Wright continued to develop this view through his entire career, and provided a further comprehensive exposition towards the very end of his life, in a paper in 1982. Wade \& Goodnight's 1991 experimental study of flour beetles purported to provide evidence of the shifting balance, but Coyne et al. 1997 countered that it did not actually test the theory. Indeed, in a very comprehensive and convincing review of theory and empirical data, Coyne et al. argued that the shifting balance was highly implausible both in theory and in practice. Their paper remains perhaps the best entrypoint into this topic, although it has been largely responsible for the general abandonment of interest in the shifting balance as the $20^{\text {th }}$ Century came to a close. Coyne et al.'s paper elicited commentaries from Peck et al. 1998 and Wade \& Goodnight 1998, defending the shifting-balance on theoretical and empirical grounds, respectively, and this was met with a further rejoinder from Coyne et al. 2000. The consensus view seems to be that the shifting balance theory provides little insight into the evolution of most species and most of their adaptations, 
but Mallet's 2010 article on mimicry rings in heliconius butterflies suggests one possible scenario in which evolution has proceeded according to Wright's theory.

Wright, S. 1932. The roles of mutation, inbreeding, crossbreeding, and selection in evolution.

Proceedings of the Sixth International Congress of Genetics 1: 356-366.

Although he had briefly mentioned it in two previous papers, it was here that Wright provided the first detailed elaboration of his view that adaptive evolution occurs via a "shifting balance" in which different subpopulations stabilize different traits that are then subjected to interpopulation selection.

Wright, S. 1982. The shifting balance theory and macroevolution. Annual Reviews of Genetics 16: 119.

An overview of the shifting-balance theory, written at the end of Wright's career, putting it in its historical context, discussing its origins, and showing the various refinements in its conceptualisation over the half century from its initial formulation.

Wade, M. J. \& Goodnight, C. J. 1991. Wright's shifting balance theory: an experimental study. Science 253: 1015-1018.

An experimental study conceived as a test of the shifting-balance theory, in which the response to selection - in terms of improved fitness - in laboratory populations of flour beetles was shown to be greatest in those treatments involving structured as opposed to randomly-mixing populations.

Coyne, J. A., Barton, N. H. \& Turelli, M. 1997. A critique of Sewall Wright's shifting balance theory of evolution. Evolution 51: 643-671.

A comprehensive review of the theoretical and empirical support - or, rather, lack thereof - for the shifting-balance theory, concluding that it is theoretically implausible and empirically irrelevant, and arguing that experiments such as Wade \& Goodnight's do not actually test the theory.

Peck, S. L., Ellner, S. P. \& Gould, F. 1998. A spatially explicit stochastic model demonstrates the feasibility of Wright's shifting balance theory. Evolution 52: 1834-1839.

A response to Coyne et al, providing a theoretical analysis of all three stages of the shiftingbalance model of evolution, and concluding that Wright's view of adaptive evolution is theoretically plausible.

Wade, M. J. \& Goodnight, C. J. 1998. The theories of Fisher and Wright in the context of metapopulations: when nature does many small experiments. Evolution 52: 1537-1553.

A response to Coyne et al., reviewing empirical evidence for the important role of epistasis and random drift in evolution, and reframing the shifting-balance theory within the wider context of the dispute between Wright and Fisher as to the importance of non-Darwinian factors in evolution.

Coyne, J. A., Barton, N. H. \& Turelli, M. 2000. Is Wright's shifting balance important in evolution? Evolution 54: 306-317.

A reply from Coyne et al. emphasising that, although epistasis and random drift certainly play a role in evolution, and that they have often been unjustly neglected, it is nevertheless the case that Wright's shifting-balance theory per se has contributed very little to our understanding of evolution.

Mallet, J. 2010. Shift happens! Shifting balance and the evolution of diversity in warning colour and mimicry. Ecological Entomology 35: 90-104. 
This paper argues that empirical data from Heliconius butterflies support the view that the evolution of aposematic warning coloration and Müllerian mimicry has proceeded in accordance with the shifting-balance theory.

\section{Cultural Group Selection}

The difficulty encountered by a strong focus on group-level selection is that this evolutionary factor is often opposed - and sometimes dwarfed - by selection acting at the within-group level, potentially rendering it relatively insignificant to evolutionary outcomes. One scenario in which heritable variation within groups may be relatively absent is in relation to cultural rather than genetical inheritance, as human groups may maintain their cultural distinctiveness in the face of significant exchange of individuals between groups that more or less homogenises them genetically. This has led to group selection enjoying relatively greater perceived utility in relation to human cultural evolution and in the social sciences more generally. An articulation of cultural evolution as an analogue of genetical evolution, and the relative importance of selection acting at the level of whole social groups and social institutions, was provided by Hayek in 1960. Apparently independently of Hayek, a research tradition on cultural evolution also grew out of population genetics, in particular with the publication of pioneering books by Cavalli-Sforza \& Feldman 1981 and Boyd \& Richerson 1985. Boyd \& Richerson 1990 later emphasised the possibility for different human groups to evolve different evolutionarily stable cultures, and for intergroup competition to select for those stable cultures that maximize group productivity, in a process very reminiscent of Wright's shifting balance (see Shifting Balance section, above). Sober \& Wilson's 1998 book makes an impassioned appeal for researchers to rethink the rejection of group selection in the 1960s and particularly targets social scientists as potential practitioners of the new group selection, especially in relation to cultural evolution. Wilson's 2002 book places even more focus on cultural evolution of human groups and explores the evolution of religion within this framework. A 2004 special issue of the Journal of Economic Behavior \& Organization devoted to cultural group selection - including a target review, 17 commentaries and a reply provides a useful overview of diversity of opinion among a number of researchers in a range of fields across the social sciences and humanities. Mesoudi's 2011 book provides a recent overview of the current state of the field of cultural evolution, with discussion of cultural group selection, and highlighting current challenges and avenues for future study.

Hayek, F. A. 1960. The constitution of liberty. London, UK: Routledge \& Kegan Paul. In chapter 4 , Hayek outlines a theory of cultural evolution, driven by imitation rather than biological inheritance, and emphasising that successful social institutions arise not by design but through a struggle for existence with other social institutions and other social groups.

Cavalli-Sforza, L. L. \& Feldman, M. W. 1981. Cultural transmission and evolution: a quantitative approach. Princeton, NJ: Princeton University Press.

This groundbreaking book formalising the rules of selection and transmission of culture in the manner of population genetics theory is mostly focused on individual-level selection, but some preliminary links to group selection are made in the Epilogue.

Boyd, R. \& Richerson, P. J. 1985. Culture and the evolutionary process. Chicago, IL: University of Chicago Press.

Another groundbreaking book on cultural evolution and its interplay with genetical evolution. The authors emphasise the potential for a cultural mode of inheritance to inflate the relative between-group variation and reduce within-group variation, promoting the response to group selection.

Boyd, R. \& Richerson, P. J. 1990. Group selection among alternative evolutionarily stable strategies. Journal of Theoretical Biology 145: 331-342. 
A theoretical paper exploring the potential for cultural group selection to promote cultural phenotypes - among the set of those that are evolutionarily stable at a within-group level that lead to greater group fitness, in a manner reminiscent of Wright's shifting balance theory of evolution.

Sober, E. \& Wilson, D. S. 1998. Unto others: the evolution and psychology of unselfish behaviour. Cambridge, MA: Harvard University Press.

Chapters 4 and 5 of this book include a useful review of theory on cultural group selection and adaptation, with application to conflicts between ancestral and contemporary human groups.

Wilson, D. S. 2002. Darwin's cathedral. Chicago, IL: University of Chicago Press.

This book explores the possibility of viewing human groups as adapted organisms in their own right, as a consequence of cultural group selection, with a particular focus on the evolution of religions.

Barkley Rosser, J. 2004. Evolution and altruism. Journal of Economic Behavior \& Organization 53: 1143.

This issue of Journal of Economic Behavior \& Organization - comprising a target article on "Cultural group selection, coevolutionary processes and large-scale cooperations", 17 commentaries and 1 reply - provides a useful overview of thinking about cultural group selection in a range of disciplines.

Mesoudi, A. 2011. Cultural evolution. Chicago, IL: University of Chicago Press.

This book provides a comprehensive and very readable overview of the current state of the study of cultural evolution, including a discussion of cultural group selection, and highlighting pressing challenges and future research directions for the field. 